\title{
Lichen sclerosus et atrophicus
}

\section{Juckreiz, Schmerzen, fleckige Herde}

\author{
VON F.-M. KöHN, M. MÖHRENSCHLAGER
}

\begin{abstract}
Schmerzen, Entzündungen und Miktionsprobleme sind nur einige der Beschwerden, die einen Lichen sclerosus et atrophicus begleiten. Die frühe Diagnose lohnt sich. Durch rechtzeitige Beschneidung können sich bei Männern die Hautveränderungen zurückbilden. Zudem haben verschiedene Topika sowie Lichttherapien ihre Wirksamkeit gezeigt.
\end{abstract}

- Der Lichen sclerosus et atrophicus ist eine chronisch entzündliche Bindegewebserkrankung der Haut, die bevorzugt (> 80\%) die Genitalregion befällt. Die Ursache ist unklar; sie kann mit einem Diabetes mellitus assoziiert sein. Zusätzlich werden traumatische Faktoren, Autoimmunerkrankungen, genetische sowie hormonelle Faktoren diskutiert.

\section{Häufigkeit}

Bei Frauen tritt Lichen sclerosus et atrophicus (LSA) fünf- bis zehnmal häufiger auf als bei Männern (meist unbeschnittene Patienten im mittleren Lebensalter). Aber auch bei Kindern ist diese Hauterkrankung schon häufig. So ergaben klinische Untersuchungen von 100 Jungen vor Zirkumzision wegen Phimose die Diagnose „Lichen sclerosus et atrophicus" in 14 Fällen [1]. Allgemein finden sich histologische Zeichen bei 3,6-19\% der Vorhäute. Im Kindesalter wird der LSA auch schon einmal als Zeichen sexuellen Missbrauchs fehlinterpretiert. Die Schwierigkeit besteht aber darin, dass sexueller Missbrauch einen LSA im Sinne eines „Koebner-Phänomens“ verstärken kann.

\section{Klinik}

Klinisch präsentiert sich die Erkrankung mit porzellanartig verfärbten, derben, zunächst kleinfleckigen, dann konfluierenden Herden, die schließlich zu größeren Flächen mit pergamentartiger Oberfläche konfluieren können (Abb. 1). Im weiteren Verlauf können auch Hämorrhagien, Erosionen und Ulzerationen auftreten. Beim Mann sind Adhäsionen zwischen Glans penis und innerem Vorhautblatt die Folge. Bei Frauen kommt es zur Sklerosierung der Labien mit Verengung des Introitus vaginae. Das Orificium urethrae kann sich so weit verengen, dass die Miktion behindert ist. Bei 29\% der Männer mit Urethrastrikturen fanden sich histopathologische Zeichen für einen Lichen sclerosus et atrophicus [2].

AnfänglichsinddieHautveränderungen symptomlos; später wird über Juckreiz, Brennen, verminderte Sensibilität der Glans penis und Schmerzhaftigkeit der Erektion bzw. vaginale Beschwerden berichtet. Beim Mann kommt es zur narbigen Verengung der Vorhaut und zu sekundären entzündlichen Veränderungen infolge von Rhagaden der Vorhaut bei Erektion und Geschlechtsverkehr (Abb. 2).

Immer sollte das gesamte Integument einschließlich der Analregion untersucht werden, um extragenitale Herde zu erfassen.

\section{Differenzialdiagnosen}

Die Differenzialdiagnosen des LSA umfassen Vitiligo, postinflammatorische Hypopigmentierungen sowie Narben durch Traumen oder Operationen oder das vernarbende Pemphigoid.

\section{Risiken und Prognose}

Bei chronischem Verlauf kann in den Herden ein spinozelluläres Karzinom auftreten. In Untersuchungen an 86 nicht zirkumzidierten Männern mit genitalem Lichen sclerosus et atrophicus fanden sich bei fünf Patienten histopatholo- gische Anzeichen für Malignität. Im Durchschnitt bestand die Erkrankung ca. 17 Jahre. Der Nachweis von HPV 16 gelang bei vier der fünf Patienten. Lichen sclerosus et atrophicus wurde bei 68/207 Männern mit penilen spinozellulären Karzinomen diagnostiziert [3].

\section{Therapie}

Therapeutisch sollte bei Männern die Beschneidung frühzeitig erwogen werden, da sich nach Zirkumzision selbst Hautveränderungen an der Glans penis zurückbilden können. Die lokale Applikation kortikoidhaltiger (Clobetasol)Cremes hat sich als effektiv erwiesen. Alternativ können Externa mit Immunmodulatoren (Tacrolimus) erwogen werden. Die früher regelmäßig empfohlenen testosteronhaltigen Cremes haben in den meisten Studien keine hinreichende Wirksamkeit gezeigt. Eine Abtragung einzelner Herde mit dem $\mathrm{CO}_{2}$-Laser ist möglich. Bei Superinfektion können auch antimikrobiell wirksame Externa empfohlen werden.

Positive Berichte über eine Lichttherapie mit UVA bei extragenitalem LSA und eigene Erfahrungen lassen auch diese Therapieoption für genitale Veränderungen Erfolg versprechend erscheinen.

\section{Literatur unter www.mmw.de}

Für die Verfasser:

Prof. Dr. med. F.-M. Köhn

Andrologicum München

Burgstr. 7, D-80331 München

Tel.: 089/29160655, Fax: 089/29160677

E-Mail: info@andrologicum.com

Koautor: Dr. med. Matthias Möhrenschlager Klinik und Poliklinik für Dermatologie und Allergologie am Biederstein, TU München

\section{Keywords}

Lichen sclerosus et atrophicus

Autoimmune-related disease Dermatosis - Chronic sclerotic process - Diagnosis - Therapy 